\title{
ЯДЕРНА ФІЗИКА
}

\author{
Francesco Vissani ${ }^{1,2, *}$ \\ ${ }^{1}$ INFN - Laboratori Nazionali del Gran Sasso, Assergi (AQ), Italy \\ ${ }^{2}$ Gran Sasso Science Institute, L'Aquila (AQ), Italy \\ *Corresponding author: francesco.vissani@lngs.infn.it
}

\section{JOINT ANALYSIS OF BOREXINO AND SNO SOLAR NEUTRINO DATA AND RECONSTRUCTION OF THE SURVIVAL PROBABILITY}

Solar neutrino oscillations are supported by KamLAND's antineutrino measurements, but certain solar neutrino data - the observed shape of the ${ }^{8} \mathrm{~B}$ flux and the difference between day and night counting rates measured in Super-K - do not fit well with the ensuing oscillation pattern. Interestingly, other solar neutrino data allow independent tests of the survival probability. Thanks to the new measurements of Borexino at low-energies along with the standard solar model and to the results of SNO at high-energies, four values of the neutrino survival probability are known. We build and study a likelihood based only on these solar neutrino data. The results agree well with the standard oscillation pattern and in particular with KamLAND findings. A related and straightforward procedure permits to reconstruct the survival probability of solar neutrinos and to assess its uncertainties, for all solar neutrino energies.

Keywords: solar neutrinos, neutrino oscillations, nuclear astrophysics, pp neutrinos, pep neutrinos, ${ }^{7} \mathrm{Be}$ neutrinos, ${ }^{8} \mathrm{~B}$ neutrinos.

\section{Introduction}

Solar neutrinos continue to provide valuable occasions of research to experimentalists and theorists working in astrophysics and in particle physics. In the present work, we aim at a fresh assessment of solar neutrino oscillations and at reconstructing the survival probability, by exploiting the new experimental results made available by Borexino Collaboration [1]. In the rest of this Section, we describe in greater detail the underlying context and motivations.

The MSW theory of neutrino oscillations [2] is widely considered reliable and consistent with other facts, e.g., with SNO neutral current results [3] and with KamLAND terrestrial antineutrino measurements [4]. However, in a recent paper of SuperKamiokande Collaboration one reads that [5], there is still no clear evidence that the solar neutrino flavor conversion is indeed due to neutrino oscillations and not caused by another mechanism.

Indeed, the measurements of ${ }^{8} \mathrm{~B}$ neutrinos of Super-Kamiokande [5] and SNO [3] are consistent with a constant suppression of the expected flux. Super-Kamiokande Collaboration [5] finds a hint for day-night effect but does not see evidence of a decrease with the energy of the survival probability (in solar neutrino jargon, "upturn" means commonly a negative and measurable value of the slope at ${ }^{8} \mathrm{~B}$ energies). SNO results [3] do not contradict these results even if they are less significant.

These results favor values of the oscillation parameter $\Delta m_{21}^{2}$ that are smaller and $2 \sigma$ away from those pointed out by the global analyses, see e.g., [6 - 8], that are mostly due to KamLAND and not by solar neutrino data themselves. More solar neutrino data are necessary to settle the issue.
In fact, this situation has stimulated the theoretical debate and new physics scenarios have been proposed, see e.g., [9, 10], and also [11] for a recent assessment.

Here, we extract the parameters of MSW theory using other and independent solar neutrino data. We include in the analysis the counting rates of 4 different branches of the solar neutrinos, measured by SNO and Borexino, the latter just appeared and not yet used in global analyses. The KamLAND results, the results on day-night asymmetry, the spectral shape of the ${ }^{8} \mathrm{~B}$ neutrinos, will not be used instead. In this manner, the results of our analysis of solar neutrino data can be compared with the other ones, verifying the consistency; as we will see, the results agree very well with the global fits and in particular with KamLAND ${ }^{1}$. We show how to use this type of analysis to reconstruct the survival probability quite precisely.

\section{The MSW survival probability}

The survival probability of electron neutrinos from the Sun, that includes three flavor effects, can be conveniently approximated as,

$$
\begin{aligned}
& P\left(E_{v} ; \Delta m_{21}^{2}, \theta_{12}\right)=\cos ^{4} \theta_{13} \times \\
\times & P_{2 \mathrm{f}}\left(E_{v} ; \Delta m_{21}^{2}, \theta_{12}\right)+\sin ^{4} \theta_{13},
\end{aligned}
$$

${ }^{1}$ The results of Homestake, Gallex/GNO and SAGE are relevant for the current global fits. However, we do not include these integral measurements (= that sum the contributions of various solar neutrino branches) since they cannot be directly attributed to a specific energy.

(C) Francesco Vissani, 2017 
where $\sin ^{2} \theta_{13} \approx 0.022$ is well-known and will be kept fixed in the analysis. To simplify the notation of the theoretical (true) survival probability $P$, we do not use any superscript or subscript; instead, we will use a subscript to identify the experimental values $P_{i}$, discussed just below. This probability depends slightly upon whether the neutrinos are detected on day or on night; however, for the region of parameters in which we are interested, this effect at most $\%$, and it is much smaller for energies below the ones of ${ }^{8} \mathrm{~B}$ neutrinos. We will consider the average survival probability between day and night,

$$
P_{2 \mathrm{f}}=\frac{1}{2}\left(P_{2 \mathrm{f}}^{\text {day }}+P_{2 \mathrm{f}}^{\text {night }}\right)
$$

and we evaluate the theoretical expression of the probability at a fixed energy. The standard twoflavor formulae are adopted, namely, $P_{2 \mathrm{f}}^{\text {day }}=\frac{1}{2}\left(1+\cos 2 \theta_{12} \cdot \cos 2 \theta_{12}^{m}\right)$ and $P_{2 \mathrm{f}}^{\text {night }}=P_{2 \mathrm{f}}^{\text {day }}+$ $+\operatorname{reg}\left(E_{v} ; \Delta m_{21}^{2}, \theta_{12}\right)$; the matter mixing angle $\cos 2 \theta_{12}^{m}\left(E_{v} ; \Delta m_{21}^{2}, \theta_{12}\right)$ and the regeneration function $\operatorname{reg}\left(E_{v} ; \Delta m_{21}^{2}, \theta_{12}\right)$ are evaluated analyticcally with the expressions summarized in [11]. We do not separate day and night data and we do not use the information on the shape of the ${ }^{8} \mathrm{~B}$ neutrino spectrum for our analysis: stated otherwise, the hint for day-night asymmetry and the (lack of) upturn at lower energies will be regarded as independent data, that lead to independent conclusions.

Illustration. To summarize and for the purpose of illustration, we show in Fig. 1 the two-flavor survival probabilities given in [11] for parameters consistent with the KamLAND observations, $\Delta m_{21}^{2}=7.4 \cdot 10^{-5} \mathrm{eV}^{2}$ and with Super-Kamiokande observations alone, $\Delta m_{21}^{2}=4.9 \cdot 10^{-5} \mathrm{eV}^{2}$. When $\Delta m_{21}^{2}$ decreases, the ${ }^{8} \mathrm{~B}$ neutrinos enter deeper into the MSW region and get further away from the transition region; thus, at ${ }^{8} \mathrm{~B}$ energies, both regeneration effect and the slope of the curve increase.

Also another (evident) effect occurs: the values of the survival probability at other energies change. In the present work, we will exploit this remark to determine the parameters of solar neutrino oscillations and to reconstruct the shape of the survival probability, since, as a matter of fact, solar neutrinos have been observed at 'high-energy', $\sim 10 \mathrm{MeV}$ ( ${ }^{8} \mathrm{~B}$ neutrinos) but also at 'low-energy' region, $\sim 1 \mathrm{MeV}$ ( $p p,{ }^{7} \mathrm{Be}$, pep neutrinos), that has been emphasized in Fig. 1.
Effects of different $\Delta \mathrm{m}^{2}$ best-fit values

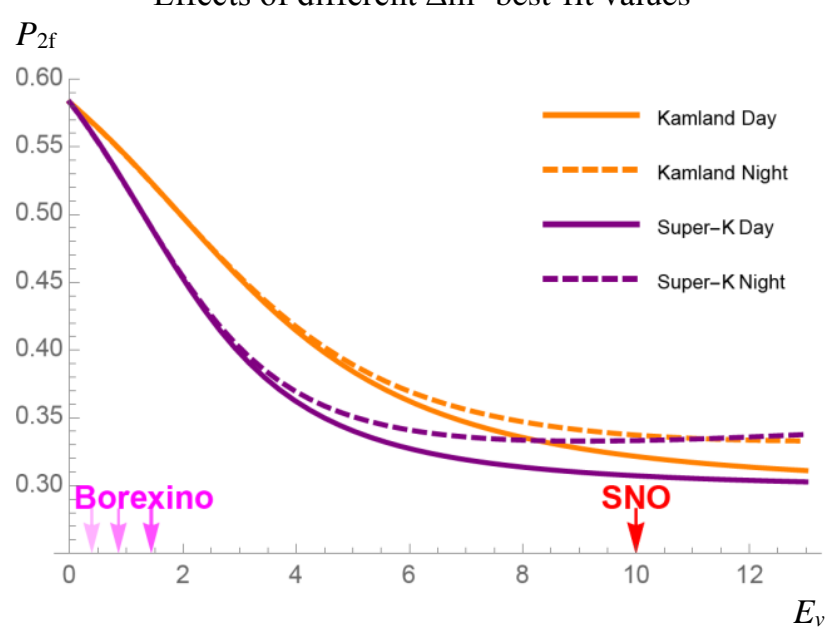

Fig 1. Two-flavor survival probabilities curves, from [11], for neutrinos produced in the center of the Sun and shown separately for those that arrive by day and those that arrive by night. The superimposed, colored arrows indicate the energies of the $p p$-branches directly observed by Borexino and SNO. (See color Figure on the journal website.)

Low energy behavior of the various survival probabilities. The various types of neutrinos are produced in different regions of the Sun; this fact matters for an accurate description of the survival probabilities of electron neutrinos. In order to show the point most clearly, we consider the low energy regime, $E_{v} \sim 1 \mathrm{MeV}$, when the corrections due to MSW theory are small and the regeneration function (due to terrestrial matter density) is even smaller. In this regime, and using the notations of [11], we note that there is a small parameter,

$$
\epsilon_{\Theta} \approx 1.04\left(\frac{n_{e}^{\odot}}{100 \mathrm{~mol}}\right) \cdot\left(\frac{7.37 \cdot 10^{-5} \mathrm{eV}^{2}}{\Delta m_{21}^{2}}\right) \cdot\left(\frac{E_{v}}{5 \mathrm{MeV}}\right) .
$$

Then we can Taylor-expand the oscillations probabilities in $\epsilon_{\odot}$, finding,

$$
P_{2 \mathrm{f}}^{\mathrm{day}}=\left(1-\frac{\sin ^{2} 2 \theta_{12}}{2}\right)-\frac{\cos 2 \theta_{12} \sin ^{2} 2 \theta_{12}}{2} \cdot \epsilon_{\odot}+\mathcal{O}\left(\epsilon_{\odot}^{2}\right),
$$

where the first term is the usual expression of the vacuum survival probability, and the second one is contributed by the MSW theory. Averaging Eq. (4) over the region of neutrino production, the electron densities get replaced by their average values $\left(n_{e, i}^{\odot}\right)$, that are different for different neutrino species $i$. Their values can be calculated by means of the standard solar model. Using [12] and adopting the version with $\mathrm{OP}$ opacities we find, 


$$
\begin{gathered}
\left\langle n_{e, i}^{\odot}\right\rangle=61.8,67.8,81.1,89.6 e^{-} \mathrm{mol} \\
\text { for } i=p p, \text { pep, }{ }^{7} \mathrm{Be},{ }^{8} \mathrm{~B} .
\end{gathered}
$$

(To be sure, the neutrinos that are produced in deeper regions have a bigger $\left\langle n_{e}^{\odot}\right\rangle$; thus, their survival probability decreases a bit faster with increasing energies.)

For example, the survival probability of $p p$ neutrinos can be approximated as,

$$
P_{2 \mathrm{f}}^{\mathrm{day}}=0.583\left(1-1.6 \% \cdot \frac{E_{\mathrm{v}}}{0.42 \mathrm{MeV}}\right)
$$

where, for this numerical evaluation, we use the oscillation parameters at the current best fit point. The corresponding distortion of the $p p$ neutrino spectrum is small but potentially interesting for future, very precise measurements.

Two last remarks are in order: 1) the survival probability that is usually shown and discussed is the one that concerns ${ }^{8} \mathrm{~B}$ neutrinos, namely, those that have been studied by Super-Kamiokande and SNO; 2) the survival probabilities, measured using $p p$, pep or ${ }^{7} \mathrm{Be}$ neutrinos at certain energies, are slightly larger than the survival probability of ${ }^{8} \mathrm{~B}$ neutrinos measured at the same energies. This is conceptually interesting even if the difference is just percent (as will be quantified more precisely later). These remarks will be relevant, in particular, for the discussion of Section 6.

\section{Expectations for the solar neutrino fluxes}

In order to investigate the transformations of solar neutrinos, and in particular those attributable to the MSW theory discussed just above, expectations on the fluxes before neutrino oscillations are necessary. The basic tool for this purpose is the standard solar model (SSM) originally developed by J. Bahcall more than half a century ago, tested and improved in the course of the years by him and by many collaborators. The residual uncertainties of the model depend upon several factors, including nuclear physics, opacities, and solar abundances of $Z>2$ elements ('metallicity'). Among the ways to validate the SSM, the main one to date remains the observation and interpretation of helioseismic p-modes.

The most recent and accurate version of the SSM to date is documented in [13]. The two models for solar abundances used there lead to significantly different predictions for helioseismic observations: the one with higher metallicity, called there B16-GS98, compares reasonably well with these observations; the other one with low metallicity does not [13]. For this reason, we adopt the expectations of the former version of the SSM for the $p p$ and for the pep-neutrinos, $\Phi_{p p}^{\odot}=\Phi_{p p}^{\mathrm{B} 16-\mathrm{GS} 98}$ and $\Phi_{p e p}^{\odot}=\Phi_{p e p}^{\mathrm{B} 16-\mathrm{GS} 98}$.

For what concerns the flux of ${ }^{8} \mathrm{~B}$ neutrinos, it is important to emphasize that this has been measured directly by SNO experiments with neutral current reactions [3]. The determination of $\mathrm{SNO}$ is compatible but more precise than the one that is provided us by the current version of the SSM, as can be seen by the following comparison,

$$
\begin{gathered}
\Phi_{\mathrm{B}}^{\mathrm{B} 16-\mathrm{GS} 98}=5.46(1 \pm 0.12) \cdot 10^{6} /\left(\mathrm{cm}^{2} \cdot \mathrm{s}\right), \\
\Phi_{\mathrm{B}}^{\mathrm{SNO}}=5.25(1 \pm 0.04) \cdot 10^{6} /\left(\mathrm{cm}^{2} \cdot \mathrm{s}\right) .
\end{gathered}
$$

It is convenient to use the flux of ${ }^{8} \mathrm{~B}$ neutrino observed by SNO, rather than the theoretical SSM prediction: $\Phi_{\mathrm{B}}^{\odot}=\Phi_{\mathrm{B}}^{\mathrm{SNO}}$. The key point is just that this expectation has nothing to do with oscillations (to be tested later); let us repeat that its advantage is that it implies an uncertainty smaller than the one of SSM.

Finally, we discuss the ${ }^{7} \mathrm{Be}$ neutrinos. Their flux depends upon the production reaction ${ }^{3} \mathrm{He}+{ }^{4} \mathrm{He} \rightarrow$ ${ }^{7} \mathrm{Be}+\gamma$, whose cross section is proportional and it is expressed in terms of the parameter $S_{34}$ (i.e., the $S$-factor, see e.g., [14] for the definition). Several extrapolations to solar energies of the available data on ${ }^{3} \mathrm{He}+{ }^{4} \mathrm{He} \rightarrow{ }^{7} \mathrm{Be}+\gamma$ are present in the literature [15], model based, and [16] based on $R$-matrix. The values of $S_{34}$ obtained in [15] and [16] are consistent within uncertainties. However, in [16], the experimental data, including elastic scattering phase shifts, are consistently described over a wider energy range. Thus at the present status of knowledge, the determination of $S_{34}$ obtained in [16] can be presumed to be more robust, as argued there and further explained in [17]. Therefore, this will be used in the following. This implies a slight change of certain SSM fluxes and more precisely a downward shift of the ${ }^{8} \mathrm{~B}$ and of the ${ }^{7} \mathrm{Be}$ fluxes by 2.7 and $2.8 \%$ respectively [13]; note incidentally that this improves even further the agreement of the central values in Eq. (7). In view of these considerations, we will apply the predicted $2.8 \%$ downward renormalization of the ${ }^{7} \mathrm{Be}$ flux; on top of that, we assume conservatively the same error $^{2}$ of the high metallicity model,

$$
\Phi_{\mathrm{Be}}^{\odot}=\Phi_{\mathrm{Be}}^{\mathrm{B} 16-\mathrm{GS} 98}(1-0.028)
$$

\footnotetext{
${ }^{2}$ It is plausible that, if the predictions of the SSM are enhanced by anchoring the ${ }^{8} \mathrm{~B}$ flux to the value measured by SNO, this will have also other impacts on the predictions, including a reduction of the uncertainty of ${ }^{7} \mathrm{Be}$ flux; however, this goes beyond the scope of the present discussion.
} 
a small revision (improvement) of the nominal value of the flux from the B16-GS98 version of the SSM, that is largely within theoretical errors. Note that by adopting this smaller value leads to increase the value of the survival probability measured by Borexino by the same factor $1 /(1-0.028)$, see next Section.

Summarizing, the expectations for the solar neutrino fluxes that we adopt are,

$$
\begin{aligned}
& \Phi_{p p}^{\odot}=(5.98 \pm 0.04) \cdot 10^{10} /\left(\mathrm{cm}^{2} \cdot \mathrm{s}\right), \\
& \Phi_{\mathrm{Be}}^{\odot}=(4.79 \pm 0.29) \cdot 10^{9} /\left(\mathrm{cm}^{2} \cdot \mathrm{s}\right), \\
& \Phi_{p e p}^{\odot}=(1.44 \pm 0.01) \cdot 10^{8} /\left(\mathrm{cm}^{2} \cdot \mathrm{s}\right), \\
& \Phi_{\mathrm{B}}^{\odot}=(5.25 \pm 0.20) \cdot 10^{6} /\left(\mathrm{cm}^{2} \cdot \mathrm{s}\right),
\end{aligned}
$$

where the numerical values of the fluxes of the $p p$ and pep neutrinos are from high metallicity SSM (version B16-GS98), as given in Table 5 of [13], and the other two are discussed above.

\section{Known values of the survival probability}

A straightforward strategy to reconstruct the pattern of solar neutrino oscillations is to constrain the survival probability of electron neutrinos using the measurements that have been obtained at various energies. This approach is possible using the results of those detectors, capable to isolate the individual branches of solar neutrinos, i.e., to measure some parts of the differential neutrino spectrum. These are Kamiokande, Super-Kamiokande, SNO, KamLAND and Borexino.

Kamiokande, Super-Kamiokande, SNO and Borexino measured the electronic neutrinos from the ${ }^{8} \mathrm{~B}$ branch; Super-Kamiokande and SNO attained the highest precision. SNO (as discussed above) measured also the total flux of neutrinos and thanks to these measurements, the suppression of the flux of the electronic neutrinos from the ${ }^{8} \mathrm{~B}$ has a special status: it is proved experimentally. Borexino and subsequently KamLAND measured neutrinos from the beryllium line, the former experiment attaining a great precision; finally, Borexino probed also the $p p$ and the pep branches. Thus, there are 4 measurements at different energies.

The formulae for the expected numbers of events (due to neutral and charged current on deuterium at SNO and due to elastic scattering in all detectors) help to clarify how it is possible to measure the (average) survival probability, knowing the SSM prediction for a certain flux $\Phi_{i}^{\text {SSM }}$, when the individual contribution can be tagged experimentally. These expressions are,

$$
\begin{aligned}
N_{\mathrm{B}}^{\mathrm{vD}, \mathrm{nc}} & =\mathcal{N}_{D} T \int d E_{v} \epsilon^{\mathrm{nc}}\left(E_{v}\right) \cdot \Phi_{\mathrm{B}}^{\mathrm{SSM}} \cdot \sigma^{\mathrm{nc}}\left(E_{\mathrm{v}}\right) \equiv N_{\mathrm{vD}, \mathrm{nc}}^{\mathrm{SSM}}, \\
N_{\mathrm{B}, \mathrm{vAc}}^{\mathrm{vAcc}} & =\mathcal{N}_{D} T \int d E_{v} \epsilon^{\mathrm{cc}}\left(E_{\mathrm{v}}\right) \cdot \Phi_{\mathrm{B}}^{\mathrm{SSM}} \cdot P_{\mathrm{B}} \cdot \sigma^{\mathrm{cc}}\left(E_{v}\right) \equiv P_{\mathrm{B}} \cdot N_{\mathrm{vD}, \mathrm{cc}}^{\mathrm{SSM}}, \\
N_{i}^{\mathrm{ES}} & =\mathcal{N}_{e} T \int d E_{v} \epsilon^{\mathrm{ES}}\left(E_{v}\right) \cdot \Phi_{i}^{\mathrm{SSM}} \cdot\left[P_{i} \cdot \sigma^{v_{e}}\left(E_{v}\right)+\left(1-\mathrm{P}_{i}\right) \cdot \sigma^{v_{\mu}}\left(E_{v}\right)\right] \equiv \\
& \equiv P_{i} \cdot\left(N_{v_{e}}^{\mathrm{SSM}}-N_{v_{\mu}}^{\mathrm{SSM}}\right)+N_{v_{\mu}}^{\mathrm{SSM}} \quad \text { with } i=p p,{ }^{7} \mathrm{Be}, p e p,{ }^{8} \mathrm{~B},
\end{aligned}
$$

where $\mathcal{N}_{\mathrm{D}}\left(\right.$ resp., $\left.\mathcal{N}_{\mathrm{e}}\right)$ is the number of deuterons (resp., of electrons), namely, of targets; $T$ is the time of measurement; $\epsilon$ are the efficiency functions; $\sigma$ the cross sections. We consider the values of $P_{i}$ averaged between day and night (assuming that the detector efficiency is constant). For neutrinos from electron capture (monochromatic), the energy is well known; for neutrinos from continuous distributions, we consider the average energy of the distributions. E.g., for Super-Kamiokande and with a threshold of 4.5 MeV, the average is at $9 \mathrm{MeV}$ whereas for $\mathrm{SNO}$ the average energy is at $10 \mathrm{MeV}$ [3]; the widths are in both cases few $\mathrm{MeV}$.

High energy. The ${ }^{8} \mathrm{~B}$ neutrinos, measured again at $\mathrm{SNO}$ with charged currents, $\Phi_{\mathrm{B}}^{v_{e}, \mathrm{SNO}}=(1.735 \pm$ \pm 0.090 ) in units of $10^{6} \mathrm{~cm}^{-2} \mathrm{~s}^{-1}$, combining phase I and phase II values [3], can be compared with the one measured by neutral currents. The ratio gives directly the value of the survival probability

$$
P_{\mathrm{B}}=\frac{\Phi_{\mathrm{B}}^{v_{e}, \mathrm{SNO}}}{\Phi_{\mathrm{B}}^{\mathrm{SNO}}}=0.33 \pm 0.02
$$

This procedure is advantageous. The charged current and neutral current have similar cross sections and the measurements are obtained with the same detector, thus, one may expect that some systematics cancel in the ratio. This value is very precise, the relative uncertainty being just $6 \%$. Interestingly, the main error $(\sim 5 \%)$ comes from the charged current measurement.

It is possible to validate this result as follows. The SNO collaboration has also obtained a fit of the day and night energy spectra; in view of our goals 
and of other considerations ${ }^{3}$, we use this analysis only as a test. Consider the average values over energy at $E_{\mathrm{B}}=10 \mathrm{MeV}$ as given in [3]. The probability of survival on day time is $c_{0}=0.317$ and the average night-day asymmetry is $a_{0}=0.046$. Thus, the average between day and night is $P_{\mathrm{B}}=$ $=\left(P^{\text {night }}+P^{\text {day }}\right) / 2=c_{0} /\left(1-a_{0} / 2\right)=0.324 \pm 0.020$, where the statistics (dominating) and systematics errors are included. This is consistent with the result derived above, that will be adopted for the following calculations.

Low energy. Three low energy branches of the $p p$ chain, namely the beryllium line at $862 \mathrm{keV}$, the fundamental $p p$ branch, and the tightly connected pep line, have been all measured precisely by Borexino [1]. The intensity of this beryllium line is known with a precision that is twice better than the SSM prediction; moreover, the observation has been confirmed by KamLAND. The $p p$ neutrinos, that are directly linked to the solar luminosity, are also measured, although with limited precision; the related pep neutrino flux is also probed, and the measurement depends slightly upon uncertain details of the SSM [1]. The best values of the survival probabilities are given directly in [1], using the B16GS98 version of the SSM [13] and including the uncertainties to the SSM. In view of the discussion of Section 3, the value of the survival probability for ${ }^{7} \mathrm{Be}$ neutrinos cited in [1], will increase by $P_{\mathrm{B}} \rightarrow$ $P_{\mathrm{B}} /(1-0.028)$ while the values of $P_{p p}$ and $P_{p e p}$ are just the same as in [1].

The known four values of the survival probability are summarized in the Table. Note that the uncertainties in the first three values include those of the SSM.

\section{The four known values of the survival probabilities}

\begin{tabular}{|c|c|c|c|c|}
\hline$i$-th solar branch & Source & Energy, MeV & Known value of $P_{i}$ & Dominant error \\
\hline$p p$ & Borexino+SSM & $\approx 0.39$ & $0.57 \pm 0.10$ & Experiment \\
\hline${ }^{7} \mathrm{Be}$ & Borexino+SSM & 0.862 & $0.545 \pm 0.05$ & Theory \\
\hline$p e p$ & Borexino+SSM & 1.442 & $0.43 \pm 0.11$ & Experiment \\
\hline${ }^{8} \mathrm{~B}$ & SNO & $\approx 10$ & $0.33 \pm 0.02$ & Experiment \\
\hline
\end{tabular}

$\mathrm{N}$ o t e. See the text for a discussion. The first three values are collectively referred to as 'low-energy' values whereas the one corresponding to the ${ }^{8} \mathrm{~B}$ neutrinos is called 'high-energy' value.

\section{Analysis of the oscillation parameters}

Method. The unambiguous measurements of the solar neutrino fluxes for several branches of the $p p$ chain, along with reliable theoretical SSM expectations, give us four values of the survival probability $P_{i} \pm \delta P_{i}$. This allows us to adopt a very direct, chi-square based procedure of analysis of the survival probability,

$$
\chi^{2}\left(\Delta m_{21}^{2}, \theta_{12}\right)=\sum_{i} \frac{\left(P\left(E_{i} ; \Delta m_{21}^{2}, \theta_{12}\right)-P_{i}\right)^{2}}{\delta P_{i}^{2}} .
$$

A more complete notation for the true survival probability would be, $P\left(E_{i} ; \Delta m_{21}^{2}, \theta_{12}, \theta_{13} ; n_{e, i}^{\odot}\right)$, but the mixing angle $\theta_{13}$ is measured very precisely by terrestrial experiments and therefore is kept fixed in this analysis and likewise, the production densities of the neutrinos $n_{e, i}^{\odot}$ are assumed to be known

${ }^{3}$ The asymmetry $A_{e e}=2\left(P^{\text {night }}-P^{\text {day }}\right) /\left(P^{\text {night }}+P^{\text {day }}\right)$ shows a decreasing trend with the energy [3], while if it was due to regular three flavor neutrino oscillations, it should increase. precisely enough and are set to their average values ${ }^{4}$, given in Eq. (5). The index $i$ runs over the types of neutrinos that are included in the analysis. It is possible to associate this chi-square to a likelihood in the usual manner,

$$
\mathcal{L}\left(\Delta m_{21}^{2}, \theta_{12}\right) \propto \exp \left[\frac{\chi^{2}\left(\Delta m_{21}^{2}, \theta_{12}\right)}{2}\right]
$$

that is normalized to unity in the (prior) search window $10^{-5} \mathrm{eV}^{2} \leq \Delta m_{21}^{2} \leq 10^{-3} \mathrm{eV}^{2}$ and $20^{\circ} \leq \theta_{12} \leq$ $\leq 40^{\circ}$. The confidence levels of two-dimensional likelihood are analyzed by using a 2-degrees-offreedom Gaussian procedure

$$
\mathcal{L}\left(\Delta m_{21}^{2}, \theta_{12}\right) \geq(1-\text { C.L. }) \cdot \mathcal{L}_{\text {best fit }}
$$

Results. The main result of the $\chi^{2}$ analysis is given in Fig. 2. The returned best fit value of $\Delta m_{21}^{2}$ is rather close to the one obtained in the global analysis [6] (consistent with [7] and [8]) that is

${ }^{4}$ This approximation for the average survival probability allows us to reduce the computational load; it implies an error of $0.3 \%$ at $10 \mathrm{MeV}$, acceptable for our purposes and much better for energies around $\mathrm{MeV}$ (see Section 2). 


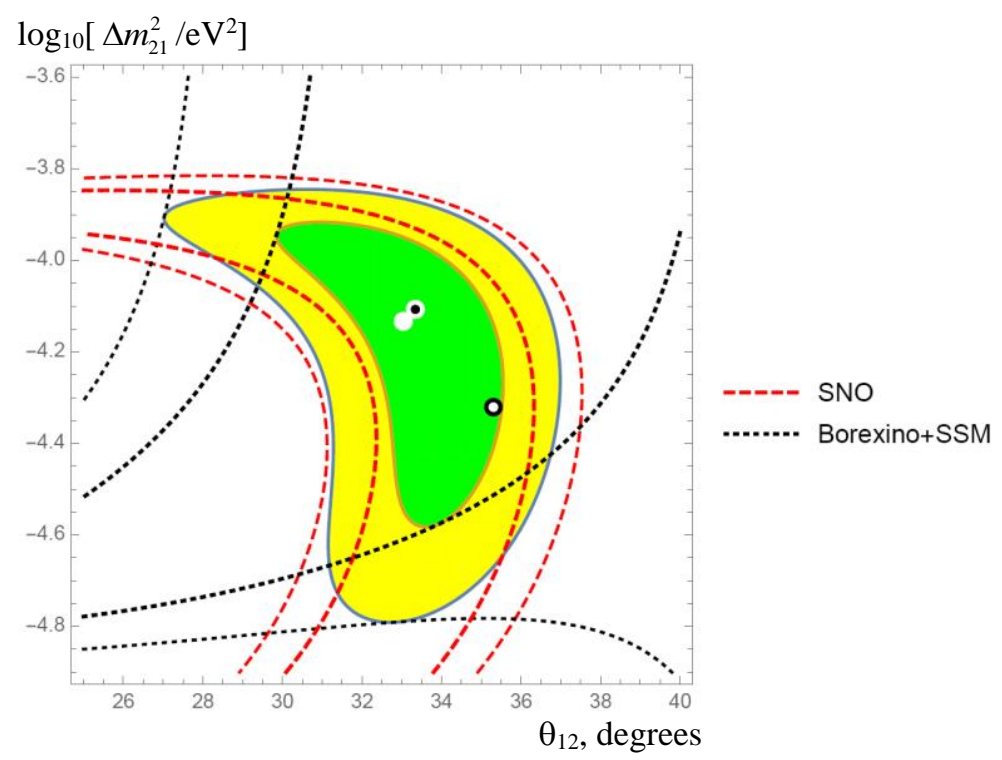

Fig. 2. The two-dimensional areas filled in green (yellow) enclose the $68.3 \%$ (95\%) confidence regions of our solar neutrino analysis. We show separately the impact of the three values of the survival probability known at low energies (Borexino+SSM, dotted lines) and of the single value known at high energy (SNO, dashed lines). The three circles show three best fit points, given in Eq. (15): the best fit point of this analysis is given by the white disk dotted in black; the white disk indicates the best global fit value; the black disk dotted in white is the best global fit of Super-Kamiokande data alone. (See color Figure on the journal website.)

driven by KamLAND findings and not by solar data, while it is somehow larger than the value indicated by Super-Kamiokande alone [5] that includes their analysis of the shape of ${ }^{8} \mathrm{~B}$ neutrinos and their measurement of the day-night asymmetry. These three values are,

\begin{tabular}{c|c|c} 
best fit & $\log _{10}\left[\Delta m_{21}^{2} / \mathrm{eV}^{2}\right]$ & $\theta_{12}$ \\
\hline this work & -4.11 & $33.4^{\circ}$ \\
global & -4.13 & $33.0^{\circ}$ \\
Super-K & -4.32 & $35.0^{\circ}$ \\
only & &
\end{tabular}

These values are displayed in Fig. 2; note that all these values are enclosed in the $1 \sigma$ (green) region of the present analysis.

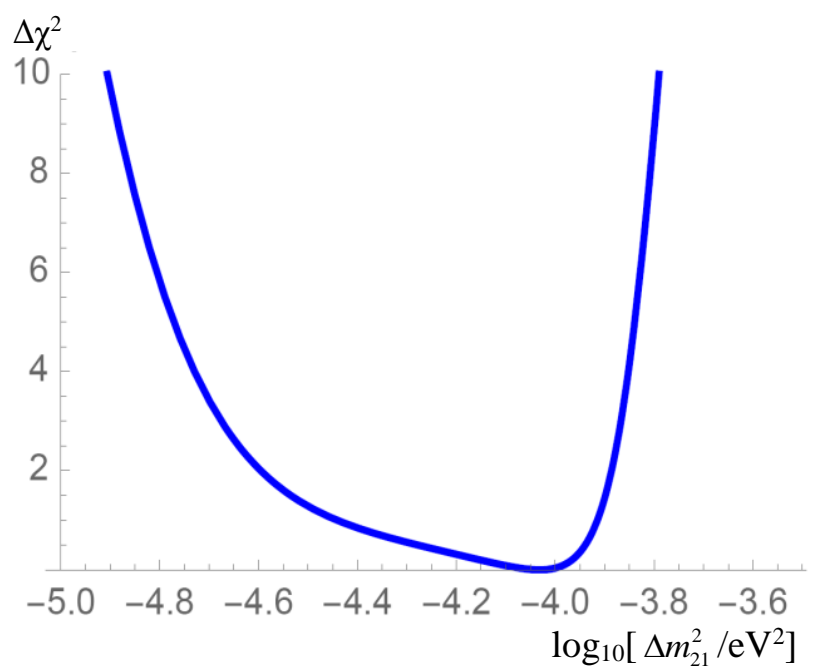

The one-dimensional $\chi_{1-\text { dof }}^{2}$ are given in Fig. 3 . These curves have been obtained setting $\chi_{1-\text { dof }}^{2}=-2 \log \mathcal{L}_{1-\text { dof, }}$ where the one-dimensional likelihood is just the full two-dimensional likelihood, integrated over the other variable (i.e., a standard marginalization procedure). The allowed ranges, that follow from the Gaussian prescription, are,

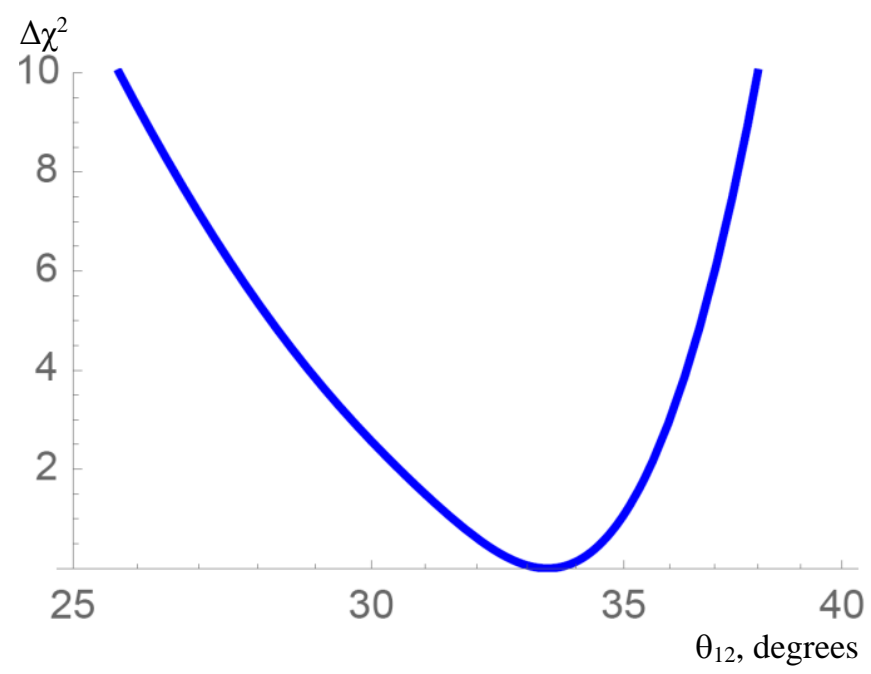

Fig. 3. One-dimensional $\Delta \chi^{2}$ distribution, for the analysis of the solar neutrino data based on the four known values of the survival probabilities summarized in the Table. 
The above ranges are compatible with those given by the global fits. In order to discuss better the meaning of these findings, let us consider the extremal $\Delta m_{21}^{2}$ values admitted at $2 \sigma$ and let us examine the position of the transition region between the vacuum and the MSW regime: for the lowest values, the ${ }^{7} \mathrm{Be}$ neutrinos fall in the transition region; instead, for the highest values, the ${ }^{8} \mathrm{~B}$ neutrinos fall in the transition region. This remark makes it evident that the above ranges are quite wide.

It is worthwhile to repeat that the best fit value of $\Delta m_{21}^{2}$ of KamLAND data is very close to the best fit range shown above, while the value of $\Delta m_{21}^{2}$ that gives an optimal fit to the Super-Kamiokande observations lies in the lowest border of the $1 \sigma$ region. Therefore, Borexino's data have some interest for the current discussion of solar neutrino findings and they indicate new ways to proceed further in the understanding of solar neutrino oscillations.

\section{Reconstruction of the survival probability}

The likelihood $\mathcal{L}\left(\Delta m_{21}^{2}, \theta_{12}\right)$ can be then used for various purposes, and in particular to reconstruct statistically the survival probability at energies different from the ones where its value is known already - i.e., to perform interpolation and extrapolation.

The most direct approach is to treat, for any value of the energy, the value of the survival probability as a random variable. Therefore, one evaluates the functions,

$$
\begin{gathered}
\left\langle P^{a}\left(E_{\mathrm{v}}\right)\right\rangle \equiv \int P^{a}\left(E_{v} ; \Delta, \theta\right) \cdot \mathcal{L}(\Delta, \theta) d \Delta d \theta \\
\text { with } a=1,2 ; \\
\delta P\left(E_{v}\right) \equiv\left[\left\langle P^{2}\left(E_{v}\right)\right\rangle-\left\langle P\left(E_{v}\right)\right\rangle^{2}\right]^{1 / 2}
\end{gathered}
$$

thereby obtaining, for each neutrino energy $E_{v}$, the average value and the range of the survival probability, that are compatible with the dataset considered. This outcome can be then compared, e.g., with the probability $P\left(E_{v} ; \Delta m_{21}^{2}, \theta_{12}\right)$ calculated at the best fit values for $\Delta m_{21}^{2}$ and $\theta_{12}$.

The resulting survival probability is shown in Fig. 4, using, for the two panels, linear and logarithmic scales. The plot in linear scale can be compared directly with Fig. 1 and emphasizes the difference between low- and high-energy measurements. The plot in logarithmic scale, instead, is often preferred in presentations of the data, e.g., [1].
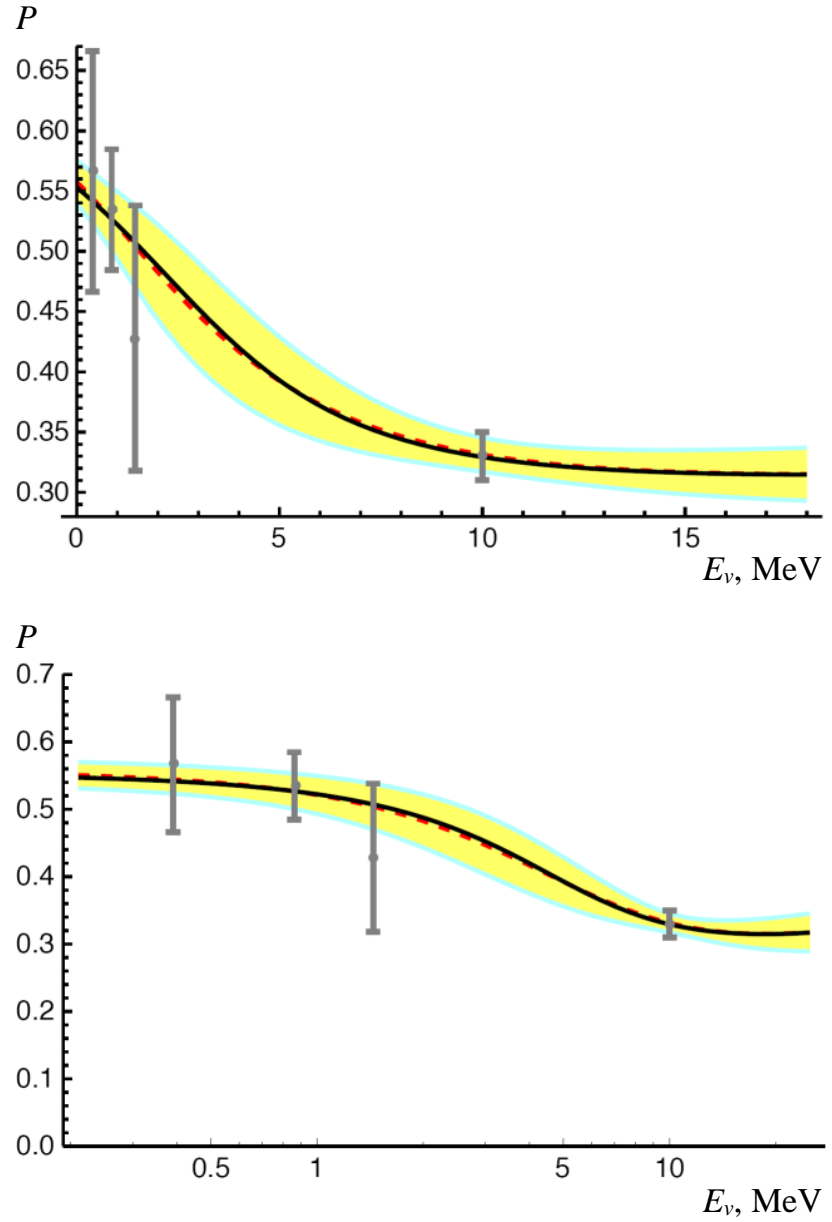

Fig. 4. The survival probability of ${ }^{8} \mathrm{~B}$ neutrinos reconstructed from the known values that have been obtained from the measured fluxes and with the help of SSM. The yellow areas enclose the $1 \sigma$ region. The data and the error-bars included are indicated by gray bars. The average probability $\left\langle P\left(E_{v}\right)\right\rangle$ (red dashed line) and the survival probability $\left\langle P\left(E_{v}\right)\right\rangle$ calculated for the best fit oscillation parameters of the present analysis (black continuous line) are also shown. Top panel: plot in linear scale. Bottom panel: plot in logarithmic scale. (See color Figure on the journal website.)

It is evident that the result of the procedure compares very satisfactorily with the known values of the survival probabilities (indicated by the vertical error-bars in gray) and that the survival probability is better constrained close to those energies where they are known, being more uncertain far from them.

In principle, a substantial improvement of the theoretical value of the beryllium line, and of the experimental measurement of the $p p$ or of the pep neutrinos, could have a big impact for the reconstruction of the survival probability: see again the Table and the discussion therein for an assessment of the dominant error.

Before concluding, let us stress that Fig. 4 shows the survival probability of the ${ }^{8} B$ neutrinos. Therefore, for consistency, the three known, central values of the survival probabilities at low energies, 
shown in the Figures by the leftmost grey points, do not coincide exactly with the values given in the Table. In fact, they are smaller by $0.7,0.4$ and $1.9 \%$ for $p p,{ }^{8} \mathrm{Be}$ and $p e p$ neutrinos respectively, as calculated at the best fit point and by using the SSM - see Section 2 for discussion.

\section{Summary and discussion}

Besides the disappearance of ${ }^{8} \mathrm{~B}$ neutrinos, there are other relevant facts that should fit into the same picture, namely the theory of three-flavor, solar neutrino oscillations. These include:

-the parameters measured by KamLAND with antineutrinos;

-the upturn of ${ }^{8} \mathrm{~B}$ neutrinos;

-the day-night asymmetry as measured with ${ }^{8} \mathrm{~B}$ neutrinos;

-the overall shape of the survival probability.

(There are also other known facts, as the measurements due to Homestake [19], SAGE [20] and Gallex/GNO [21], absence of an observable day-night asymmetry at lower energy [22], the new measurement of ${ }^{8} \mathrm{~B}$ neutrinos with a very low threshold [23]; in future, perhaps, also the shape of the $p p$ neutrinos and the intensity and shape of $\mathrm{CNO}$ neutrino flux could be measured.)

To date, there is a bit of tension between the first three aspects. No simple way out is known within the conventionally accepted physics framework: in principle, one may object that the first measurement concerns reactor antineutrinos and not solar neutrinos but the standard theory predicts that $\Delta m_{21}^{2}$ is the same for neutrinos and antineutrinos. Moreover, the shape of the reactor neutrinos does not seem to need radical revisions; the shape of ${ }^{8} \mathrm{~B}$ neutrinos may be uncertain but only within percent $[24,25]$; the day-night asymmetry seems to be even less unambiguous to interpret than the rest.

Therefore, in this work, we focused on the last item of the above list, exploiting the precise measurements, obtained very recently by Borexino, of three branches of the $p p$-chain at low energy along with SNO measurements.
We used a very simple and transparent procedure that moreover is adequate for the task; indeed, the main limitation of this analysis is just the precision of the current knowledge of the input values of the survival probability. We checked the stability of our findings under many types of variations, e.g., omitting $p p$ and/or pep data-point, using the nominal $\mathrm{SSM}$ prediction for the ${ }^{7} \mathrm{Be}$ [13], etc. The only relatively major aspect is the inclusion of the neutral current measurement of SNO.

We showed that the existing measurements of the differential flux from 4 branches of the $p p$-chain allow us to obtain the oscillation parameters, whose values are in good agreement with those measured by KamLAND. We indicated how to reconstruct very directly the overall shape of the survival probability, estimating its uncertainties.

We emphasized that the standard solar model remains important for the prediction. Indeed, the most precise measurement of Borexino, the beryllium line, is also the one for which the knowledge of the survival probability is limited by theory and not by the rate observed by Borexino. Diminishing the current theoretical uncertainty can have an important impact on the current discussion.

On the other hand, it is possible at least in principle to proceed experimentally in the measurement of the $p p$ (and partly of the pep, in view of the $\mathrm{CNO}$ neutrinos) and to obtain more precise values of the survival probability, remaining free from theoretical limitations.

I thank G. Ranucci for the conversation that triggered the present investigation [26]. I am grateful to G. Bellini and to the participants in the workshop Recent Developments in Neutrino Physics and Astrophysics, LNGS and GSSI, Sep. 2017 [18], and in particular to F. Calaprice, A. Di Leva, A. Ianni, C. Mascaretti, A. Palazzo, A. Yu. Smirnov and F. L. Villante for several useful discussions. Finally, I would like to thank an anonymous Referee of Nuclear Physics and Atomic Energy for a very accurate and helpful review.

\section{REFERENCES}

1. Borexino Collaboration (M. Agostini et al.). First simultaneous precision spectroscopy of $p p,{ }^{7} \mathrm{Be}$ and pep solar neutrinos with Borexino phase-II. arXiv: 1707.09279 [hep-ex], submitted for publication.

2. L. Wolfenstein. Neutrino oscillations in matter. Phys. Rev. D 17 (1978) 2369; S.P. Mikheyev, A.Yu. Smirnov. Resonant amplification of neutrino oscillations in matter and spectroscopy of solar neutrinos. Sov. J. Nucl. Phys. 42 (1986) 913.

3. SNO Collaboration (A. Bellerive et al.). The Sudbury Neutrino Observatory. Nucl. Phys. B 908
(2016) 30.

4. KamLAND Collaboration (A. Gando et al.). Reactor on-off antineutrino measurement with KamLAND. Phys. Rev. D 88 (2013) 033001.

5. Super-Kamiokande Collaboration (K. Abe et al.). Solar neutrino measurements in Super-KamiokandeIV. Phys. Rev. D 94 (2016) 052010.

6. F. Capozzi et al. Global constraints on absolute neutrino masses and their ordering. Phys. Rev. D 95 (2017) 096014.

7. I. Esteban et al. Updated fit to three neutrino mixing: 
exploring the accelerator-reactor complementarity. JHEP 01 (2017) 087.

8. P.F. de Salas et al. Status of neutrino oscillations 2017. arXiv:1708.01186 [hep-ph], submitted for publication.

9. P.C. de Holanda, A.Y. Smirnov. Solar neutrino spectrum, sterile neutrinos, and additional radiation in the Universe. Phys. Rev. D 83 (2011) 113011.

10. A. Palazzo. Hint of nonstandard Mikheyev-SmirnovWolfenstein dynamics in solar neutrino conversion. Phys. Rev. D 83 (2011) 101701.

11. F. Vissani. Solar neutrino physics on the beginning of 2017. Nucl. Phys. At. Energy 18(1) (2017) 5.

12. J.N. Bahcall, A.M. Serenelli, S. Basu. New Solar Opacities, Abundances, Helioseismology, and Neutrino Fluxes. Astrophys. J. 621 (2005) L85.

13. N. Vinyoles et al. A New Generation of Standard Solar Models. Astrophys. J. 835 (2017) 202.

14. D.G. Yakovlev et al. Simple analytic model for astrophysical $S$ factors. Phys. Rev. C 82 (2010) 044609.

15. E.G. Adelberger et al. Solar fusion cross sections. II. The $p p$ chain and CNO cycles. Rev. Mod. Phys. 83 (2011) 195.

16. R.J. de Boer et al. Monte Carlo uncertainty of the ${ }^{3} \mathrm{He}(\alpha, \gamma)^{7}$ Be reaction rate. Phys. Rev. C 90 (2014) 035804.
17. A. Di Leva, talk at [18].

18. Int. Conf. "Recent Developments in Neutrino Physics and Astrophysics", $4-7$ Sept. 2017, Assergi, Italy.

19. B.T. Cleveland et al. Measurement of the Solar Electron Neutrino Flux with the Homestake Chlorine Detector. Astrophys. J. 496 (1998) 505.

20. SAGE Collaboration (J.N. Abdurashitov et al.). Measurement of the solar neutrino capture rate with gallium metal. III. Results for the 2002 - 2007 datataking period. Phys. Rev C 80 (2009) 015807.

21. F. Kaether et al. Reanalysis of the Gallex solar neutrino flux and source experiments. Phys. Lett. B 685 (2010) 47.

22. Borexino Collaboration (G. Bellini et al.). Absence of a day-night asymmetry in the ${ }^{7} \mathrm{Be}$ solar neutrino rate in Borexino. Phys. Lett. B 707 (2012) 22.

23. Borexino Collaboration (M. Agostini et al.). Improved measurement of ${ }^{8} \mathrm{~B}$ solar neutrinos with $1.5 \mathrm{kt} y$ of Borexino exposure. arXiv:1709.00756 [hep-ex], submitted for publication.

24. J.N. Bahcall et al. Standard neutrino spectrum from ${ }^{8}$ B decay. Phys. Rev. C 54 (1996) 411.

25. W.T. Winter et al. The ${ }^{8} \mathrm{~B}$ neutrino spectrum. Phys. Rev. C 73 (2006) 025503.

26. F. Vissani. We saw the engine of the Sun!
Ф. Вiссанi ${ }^{1,2, *}$
${ }^{1}$ Національний інститут ядерної фізики, Національна лабораторія Гран Сассо, Ассерджі, Італія
${ }^{2}$ Науковий інститут Гран Сассо, Аквіла, Італія
*Відповідальний автор: francesco.vissani@lngs.infn.it

\section{СПІЛЬНИЙ АНАЛІЗ ДАНИХ ПРО СОНЯЧНІ НЕЙТРИНО \\ ВІД ЕКСПЕРИМЕНТІВ ВOREXINO TA SNO \\ ТА РЕКОНСТРУКЦІЯ ЙМОВІРНОСТІ ЇХНЬОГО ВИЖИВАННЯ}

Існування осциляцій сонячних нейтрино підтверджується вимірюваннями потоків антинейтрино в експерименті KamLAND, але певні дані про сонячні нейтрино - спостережена форма спектра ${ }^{8} \mathrm{~B}$ та різниця між інтенсивністю денного та нічного потоків, виміряна в Super-K, - не дуже добре вписуються в результуючу осциляційну модель. Цікаво, що інші дані про сонячні нейтрино дають змогу провести незалежні тести ймовірності виживання нейтрино. Завдяки новим вимірюванням Borexino при малих енергіях разом із стандартною сонячною моделлю та результатами SNO при високих енергіях зараз відомі чотири значення ймовірності виживання нейтрино. Ми будуємо та вивчаємо математичну правдоподібність лише на основі цих даних про сонячні нейтрино. Результати добре узгоджуються зі стандартною осциляційною моделлю та, зокрема, з результатами KamLAND. Відповідна пряма процедура дає змогу реконструювати ймовірність виживання та оцінити їі невизначеність для всіх енергій сонячних нейтрино.

Ключові слова: сонячні нейтрино, нейтринні осциляції, ядерна астрофізика, рр нейтрино, рер нейтрино, ${ }^{7} В е$ нейтрино, ${ }^{8}$ В нейтрино.

\section{Ф. Виссани ${ }^{1,2, *}$}
${ }^{1}$ Национальный институт ядерной физики, Наџиональная лаборатория Гран Сассо, Ассерджи, Италия
${ }^{2}$ Научный институт Гран Сассо, Аквила, Италия
*Ответственный автор: francesco.vissani@lngs.infn.it

\section{СОВМЕСТНЫЙ АНАЛИЗ ДАННЫХ ПО СОЛНЕЧНЫМ НЕЙТРИНО \\ ОТ ЭКСПЕРИМЕНТОВ ВОRЕХINO И SNO И РЕКОНСТРУКЦИЯ ВЕРОЯТНОСТИ ИХ ВЫЖИВАНИЯ}

Существование осцилляций солнечных нейтрино подтверждается измерениями потоков антинейтрино в эксперименте KamLAND, но некоторые данные по солнечным нейтрино - наблюдаемая форма спектра ${ }^{8} \mathrm{~B}$ и разница между интенсивностью дневного и ночного потоков, измеренная в Super-K, - не очень хорошо 
вписываются в результирующую осцилляционную модель. Интересно, что другие данные по солнечным нейтрино разрешают провести независимые тесты вероятности выживания нейтрино. Благодаря новым измерениям Borexino при малых энергиях вместе со стандартной солнечной моделью и результатами SNO при высоких энергиях сейчас известны четыре значения вероятности выживания нейтрино. Ми строим и изучаем математическую правдоподобность только на основе этих данных по солнечным нейтрино. Результаты хорошо согласуются со стандартной осцилляционной моделью и, в частности, с результатами KamLAND. Соответствующая прямая процедура позволяет реконструировать вероятность выживания и оценить ее неопределенность для всех энергий солнечных нейтрино.

Ключевые слова: солнечные нейтрино, нейтринные осцилляции, ядерная астрофизика, рр нейтрино, рер нейтрино, ${ }^{7} \mathrm{Be}$ нейтрино, ${ }^{8} \mathrm{~B}$ нейтрино.

Надійшла 27.09.2017

Received 27.09.2017 